\title{
The two-phase MINLP optimization of a single-storey industrial steel building
}

\author{
T. Žula \& S. Kravanja \\ University of Maribor, Faculty of Civil Engineering, \\ Smetanova 17, 2000 Maribor, Slovenia
}

\begin{abstract}
The paper presents the two-phase MINLP topology and standard sizes optimization of a single-storey industrial steel building. The structure consists of main portal frames, connected with purlins. It is made from standard hot rolled I sections. The Mixed-Integer Non-Linear Programming (MINLP) optimization of the structure is applied. The MINLP performs a discrete topology and standard dimension optimization, while continuous parameters (stresses, deflections, mass, costs, etc.) are calculated simultaneously inside the continuous space. Since the discrete/continuous optimization problem of this type of structures is non-convex and highly non-linear, the Modified Outer-Approximation/EqualityRelaxation (OA/ER) algorithm is used for the optimization. The defined mass objective function is subjected to the set of equality and inequality constraints known from the structural analysis and dimensioning. The dimensioning of steel members is performed in accordance with Eurocode 3.

The MINLP optimization is performed in two phases. In the first phase, the topology optimization of the structure at the relaxed dimensions is performed only. This phase gives a good linear global approximation to the structure for the next phase. When the optimal topology is reached, the optimization is continued at the second phase for the overall topology and standard section discrete optimization. Alongside the optimal structure mass, the optimal topology with the optimal number of portal frames and purlins as well as all standard crosssections are obtained. The paper includes the theoretical basis and a practical example with the results of the optimization.
\end{abstract}

Keywords: optimization, topology optimization, discrete sizes optimization, mixed-integer non-linear programming, MINLP, industrial steel building. 


\section{Introduction}

Over the past thirty years, researchers developed and used many different algorithms for structural optimization. In the field of the optimization of steel skeletal structures various optimization methods have been proposed. O'Brien and Dixon [1] have proposed a linear programming approach for the optimal design of pitched roof frames. Guerlement et al. [2] have introduced a practical method for single-storey steel structures, based on a discrete minimum weight design and Eurocode 3 [3] design constraints. Recently, Saka [4] has considered an optimum design of pitched roof steel frames with haunched rafters by using a genetic algorithm. One of the latest researches reported in this field is the work of Hernández et al. [5], where the authors have considered a minimum weight design of the steel portal frames with software developed for the structural optimization. It should be noted that all the mentioned authors deal with the discrete sizes optimization at fixed structural topologies.

This paper discusses the simultaneous topology, standard sizes and continuous parameter optimization of an unbraced single-storey industrial steel building. The optimization of the portal frames and purlins was performed by the Mixed-Integer Non-linear Programming approach (MINLP). The MINLP is a combined discrete and continuous optimization technique. In this way, the MINLP performs the discrete topology (i.e. the number of frames and purlins) and the standard dimension (i.e. the standard cross-section sizes of the columns, beams and purlins) optimization simultaneously with the continuous optimization of the parameters (e.g. the structure mass, internal forces, deflections, etc.).

The MINLP discrete/continuous optimization problems of frame structures are in most cases comprehensive, non-convex and highly non-linear. The optimization is proposed to be performed through three steps. The first one includes the generation of a mechanical superstructure of different topology and standard dimension alternatives, the second one involves the development of an MINLP model formulation and the last one consists of a solution for the defined MINLP optimization problem.

The objective of the optimization is to minimize the mass of the singlestorey industrial building. The mass objective function is subjected to the set of equality and inequality constraints known from the structural analysis and dimensioning. The dimensioning of steel members is performed in accordance with Eurocode 3.

The Modified Outer-Approximation/Equality-Relaxation algorithm is used to perform the optimization, see Kravanja and Grossmann [6], Kravanja et al. [7,8].

The two-phase MINLP optimization is proposed. It starts with the topology optimization, while the standard dimensions are temporarily relaxed into continuous parameters. When the optimal topology is found, the standard dimensions of the cross-sections are re-established and the simultaneous discrete topology and standard dimension optimization of the beams, columns and purlins is then continued until the optimal solution is found. 


\section{Single-storey industrial building}

The paper presents the topology and standard sizes optimization of unbraced rigid single-storey industrial building steel structures, Fig. 1. The columns, beams and purlins are proposed to be built up of standard hot rolled steel I sections.

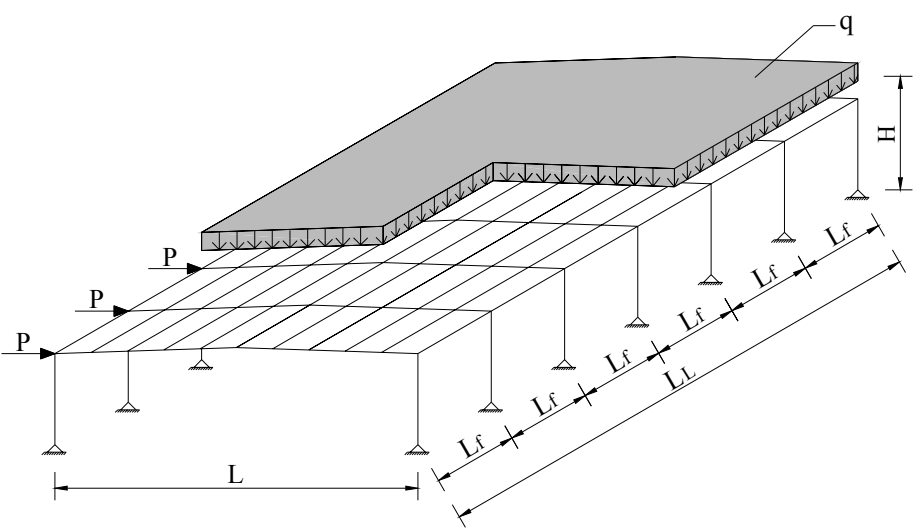

Figure 1: $\quad$ Single-storey industrial building.

The considered portal frame structures are optimized under the combined effects of the self-weight of the frame members, a uniformly distributed surface variable load (snow and wind) and a concentrated horizontal variable load (wind). The purlins are designed to transfer the permanent load (the self-weight of the purlins and the weight of the roof) and the variable load (snow and wind). The internal forces are calculated by the elastic first-order method. The dimensioning of the steel members is performed in accordance with Eurocode 3 for the conditions of both the ultimate limit state (ULS) and the serviceability limit state (SLS).

When the ULS is considered, the elements are checked for the axial, shear and bending moment resistance, for the interaction between the bending moment and the axial force, the interaction between the axial compression/buckling and the buckling resistance moment.

The total deflection $\delta_{\max }$ subjected to the overall load and the deflections $\delta_{2}$ subjected to the variable imposed load are calculated to be smaller than the limited maximum values: span/200 and span/250, respectively. The horizontal deflections are also checked for the recommended limits: the relative horizontal deflection of the portal frame should be smaller then the frame height $/ 150$.

\section{MINLP model formulation}

It is assumed that a non-convex, non-linear discrete and continuous optimization problem can be formulated as a general MINLP problem (MINLP-G) in the form: 


$$
\begin{array}{rl} 
& \min \quad z=\boldsymbol{c}^{T} \boldsymbol{y}+f(\boldsymbol{x}) \\
\text { s.t. } \quad \boldsymbol{h}(\boldsymbol{x})=\boldsymbol{0} & \boldsymbol{g}(\boldsymbol{x}) \leq \boldsymbol{0} \\
& \boldsymbol{B} \boldsymbol{y}+\boldsymbol{C} \boldsymbol{x} \leq \boldsymbol{b} \\
\boldsymbol{x} \in X= & \left\{\boldsymbol{x} \in R^{n}: \boldsymbol{x}^{L O} \leq \boldsymbol{x} \leq \boldsymbol{x}^{U P}\right\} \\
\boldsymbol{y} \in Y=\{0,1\}^{m}
\end{array}
$$

(MINLP-G)

where $\boldsymbol{x}$ is a vector of continuous variables specified in the compact set $X$ and $\boldsymbol{y}$ is a vector of discrete, mostly binary $0-1$ variables. Functions $f(\boldsymbol{x}), \boldsymbol{h}(\boldsymbol{x})$ and $\boldsymbol{g}(\boldsymbol{x})$ are non-linear functions involved in the objective function $z$, the equality and inequality constraints, respectively. All functions $f(\boldsymbol{x}), \boldsymbol{h}(\boldsymbol{x})$ and $\boldsymbol{g}(\boldsymbol{x})$ must be continuous and differentiable. All functions $f(\boldsymbol{x}), \boldsymbol{h}(\boldsymbol{x})$ and $\boldsymbol{g}(\boldsymbol{x})$ must be continuous and differentiable. Finally, $\boldsymbol{B} \boldsymbol{y}+\boldsymbol{C} \boldsymbol{x} \leq \boldsymbol{b}$ represents a subset of mixed linear equality/inequality constraints.

The above general MINLP-G model formulation has been adapted for the optimization of mechanical superstructures. The resulting MINLP formulation for mechanical superstructures (MINLP-MS) that is more specific, particularly in variables and constraints, can be used also for the modelling the steel industrial buildings. It is given in the following form:

$$
\begin{array}{cc}
\min \quad z=\boldsymbol{c}^{T} \boldsymbol{y}+f(\boldsymbol{x}) \\
\text { s.t. } \quad \boldsymbol{h}(\boldsymbol{x})=\boldsymbol{0} \\
\boldsymbol{g}(\boldsymbol{x}) \leq \boldsymbol{0} \\
\boldsymbol{A}(\boldsymbol{x}) \leq \boldsymbol{a} \\
\quad \boldsymbol{E} \boldsymbol{y} \leq \boldsymbol{e} \\
\boldsymbol{D}^{e}+\boldsymbol{R}(\boldsymbol{x}) \leq \boldsymbol{r} \\
& \boldsymbol{K}^{e}+\boldsymbol{L}\left(\boldsymbol{d}^{c n}\right) \leq \boldsymbol{k} \\
& \boldsymbol{P} \boldsymbol{y}+\boldsymbol{S}\left(\boldsymbol{d}^{S t}\right) \leq \boldsymbol{s} \\
\boldsymbol{x} \in X= & \left\{\boldsymbol{x} \in R R^{n}: \boldsymbol{x}^{L O} \leq \boldsymbol{x} \leq \boldsymbol{x}^{U P}\right\} \\
\boldsymbol{y} \in Y=\{0,1\}^{m}
\end{array}
$$

The MINLP model formulation for mechanical superstructures is proposed to be described as follows:

- Included are continuous variables $\boldsymbol{x}=\{\boldsymbol{d}, \boldsymbol{p}\}$ and discrete binary variables $\boldsymbol{y}=\left\{\boldsymbol{y}^{e}, \boldsymbol{y}^{s t}\right\}$. Continuous variables are partitioned into design variables $\boldsymbol{d}=\left\{\boldsymbol{d}^{c n}\right.$, $\left.\boldsymbol{d}^{\text {st }}\right\}$ and into performance (non-design) variables $\boldsymbol{p}$, where subvectors $\boldsymbol{d}^{c n}$ and $\boldsymbol{d}^{\text {st }}$ stand for continuous and standard dimensions, respectively. Subvectors of the binary variables $\boldsymbol{y}^{e}$ and $\boldsymbol{y}^{\text {st }}$ denote the potential existence of structural elements inside the superstructure (the topology determination) and the potential selection of standard dimension alternatives, respectively.

The mass or economical objective function $z$ involves fixed mass or cost charges in the linear term $\boldsymbol{c}^{\mathrm{T}} \boldsymbol{y}$ and the dimension dependant mass or costs in the term $f(\boldsymbol{x})$. 
- Parameter non-linear and linear constraints $\boldsymbol{h}(\boldsymbol{x})=\mathbf{0}, \boldsymbol{g}(\boldsymbol{x}) \leq \boldsymbol{0}$ and $\boldsymbol{A}(\boldsymbol{x}) \leq \boldsymbol{a}$ represent a rigorous system of the design, loading, resistance, stress, deflection, etc. constraints known from the structural analysis.

- Integer linear constraints $\boldsymbol{E} \boldsymbol{y} \leq \boldsymbol{e}$ are proposed to describe the relations between binary variables.

- $\quad$ Mixed linear constraints $\boldsymbol{D} \boldsymbol{y}^{e}+\boldsymbol{R}(\boldsymbol{x}) \leq \boldsymbol{r}$ restore interconnection relations between currently selected or existing structural elements (corresponding $\left.y^{e}=1\right)$ and cancel relations for currently disappearing or nonexisting elements (corresponding $y^{e}=0$ ).

- Mixed linear constraints $\boldsymbol{K} \boldsymbol{y}^{e}+\boldsymbol{L}\left(\boldsymbol{d}^{c n}\right) \leq \boldsymbol{k}$ are proposed to define the continuous design variables for each existing structural element. The space is defined only when the corresponding structure element exists $\left(y^{e}=1\right)$, otherwise it is empty.

- $\quad$ Mixed linear constraints $\boldsymbol{P} \boldsymbol{y}+\boldsymbol{S}\left(\boldsymbol{d}^{\boldsymbol{s t}}\right) \leq \boldsymbol{s}$ define standard design variables $\boldsymbol{d}^{s t}$. Each standard dimension $d^{s t}$ is determined as a scalar product between its vector of $i, i \in I$, discrete standard dimension constants $\boldsymbol{q}=\left\{q_{1}, q_{2}, q_{3, \ldots}, q_{i}\right\}$ and its vector of subjected binary variables $\boldsymbol{y}^{s t}=\left\{y^{s t}{ }_{1}, y^{s t}{ }_{2}, y^{s t}{ }_{3, \ldots}, y^{s t}{ }_{i}\right\}$, see Eq. 1 . Only one discrete value can be selected for each standard dimension since the sum of the binary variables must be equal to 1 (Eq. 2):

$$
\begin{gathered}
d^{s t}=\sum_{i \in I} q_{i} y_{i}^{s t} \\
\sum_{i \in I} y_{i}^{s t}=1
\end{gathered}
$$

\section{The optimization}

After the MINLP model formulation is developed, the defined MINLP optimization problem is solved by the use of a suitable MINLP algorithm and strategies. A general MINLP class of optimization problem can be solved in principle by the following algorithms and their extensions:

- the Nonlinear Branch and Bound, NBB, proposed and used by many authors, e.g. E.M.L. Beale [9], O.K. Gupta and A. Ravindran [10];

- the Sequential Linear Discrete Programming method, SLDP, by G.R. Olsen and G.N. Vanderplaats [11] and M. Bremicker et al. [12];

- the Extended Cutting Plane method by T. Westerlund and F. Pettersson [13];

- Generalized Benders Decomposition, GBD, by J.F. Benders [14], A.M. Geoffrion [15];

- the Outer-Approximation/ Equality-Relaxation algorithm, OA/ER, by G.R. Kocis and I.E. Grossmann [16];

- the Feasibility Technique by H. Mawengkang and B.A. Murtagh [17]; and - the LP/NLP based Branch and Bound algorithm by I. Quesada and I.E. Grossmann [18]. 


\subsection{Modified OA/ER algorithm}

The OA/ER algorithm consists of solving an alternative sequence of Non-linear Programming (NLP) optimization subproblems and Mixed-Integer Linear Programming (MILP) master problems. The former corresponds to continuous optimization of parameters for a mechanical structure with a fixed topology (and fixed discrete/standard dimensions) and yields an upper bound to the objective to be minimized. The latter involves a global approximation to the superstructure of alternatives in which a new topology, discrete/standard dimensions are identified so that its lower bound does not exceed the current best upper bound. The search of a convex problem is terminated when the predicted lower bound exceeds the upper bound, otherwise it is terminated when the NLP solution can be improved no more. The OA/ER algorithm guarantees the global optimality of solutions for convex and quasi-convex optimization problems.

The OA/ER algorithm as well as all other mentioned MINLP algorithms do not generally guarantee that the solution found is the global optimum. This is due to the presence of nonconvex functions in the models that may cut off the global optimum. In order to reduce undesirable effects of nonconvexities, the Modified OA/ER algorithm was proposed by Z. Kravanja and I.E. Grossmann [6], see also S. Kravanja et al. [7,8], by which the following modifications are applied for the master problem: the deactivation of linearizations, the decomposition and the deactivation of the objective function linearization, the use of the penalty function, the use of the upper bound on the objective function to be minimized as well as the global convexity test and the validation of the outer approximations.

\subsection{Two-phase MINLP optimization}

The optimal solution of a complex non-convex and non-linear MINLP problem with a high number of discrete decisions is in general very difficult to obtain. The optimization is thus proposed to be performed sequentially in two different phases to accelerate the convergence of the OA/ER algorithm. The optimization is proposed to start with the discrete topology optimization of the building, while the standard dimensions are temporarily relaxed into continuous parameters. Topology and continuous parameter optimization is soluble (a smaller combinatorial problem) and accumulates a good global linear approximation of the superstructure (a good starting point for the next phase overall optimization). When the optimal topology is found, the standard sizes of the cross-sections are re-established and the simultaneous discrete optimization of the topology and standard dimensions of the beams, columns and purlins is then continued until the optimal solution is found.

The two-phase strategy requires that the binary variables should be defined in one uniform set. In the first phase, only the binary variables which are subjected to topology alternatives become active. Binary variables of standard dimension alternatives are temporarily excluded (set on value zero) until the beginning of the second phase, in which they participate in the simultaneous overall optimization. The same holds for standard dimension logical constraints. In the 
first phase they are excluded, while the second phase includes them into the optimization.

\section{The numerical example}

The example presents the MINLP topology and standard dimension optimization of a single-storey industrial building. The building is 25 meters wide, 70 meters long and 7.5 meters heigh. The structure is consisted from equal non-sway steel portal frames which are mutually connected with the purlins.

The portal frame is subjected to self-weight $g$, uniformly distributed surface variable load $q$ (snow $s$ and wind $w$ ) and the concentrated variable load at the top of the columns $P$ (horizontal wind). Variable imposed load $s=1,8 \mathrm{kN} / \mathrm{m}^{2}$ (snow), $w_{v}=0,15 \mathrm{kN} / \mathrm{m}^{2}$ (vertical wind) and $w_{h}=0,4 \mathrm{kN} / \mathrm{m}^{2}$ (horizontal wind) are defined as the uniformly distributed surface load in the model input data. Both, the horizontal concentrated load and the vertical uniformly distributed line load on the beams are calculated considering the intermediate distance betwen the portal frames.

The portal frame superstructure was generated in which all possible structures were embedded by 30 portal alternatives, 20 purlin alternatives and different standard size variation. The superstructure comprised 24 different standard hot rolled European wide flange I beams, i.e. HEA sections (from HEA 100 to HEA 1000) for each column, beam and purlin seperately. The material used was steel S 355 .

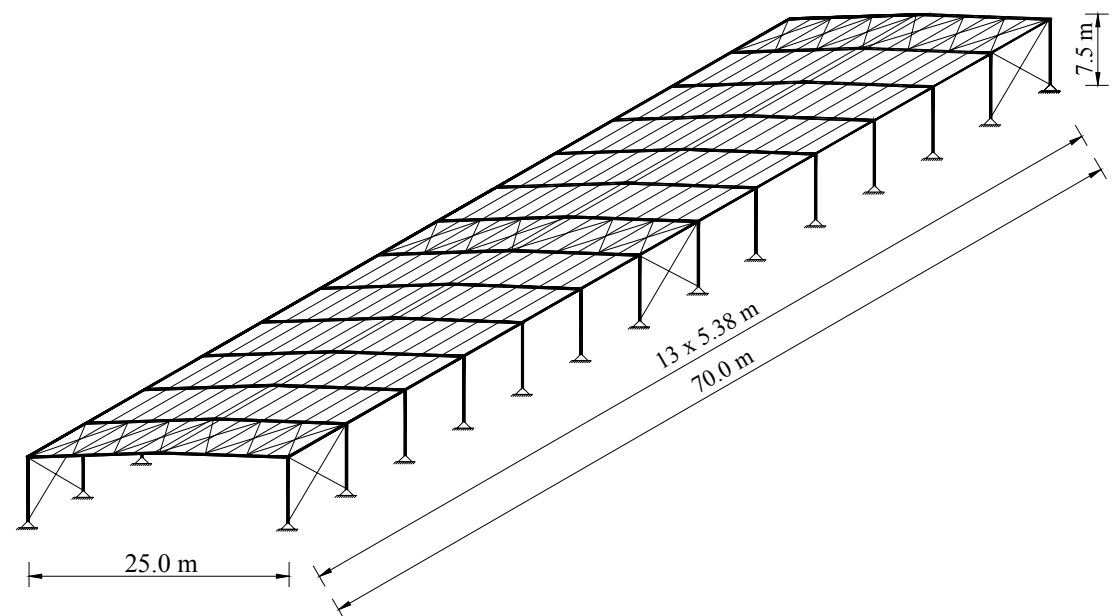

Figure 2: $\quad$ Optimal topology of the single-storey industrial building.

The optimization was carried out by a user-friendly version of the MINLP computer package MIPSYN, the successor of PROSYN [6] and TOP [19]. As an interface for mathematical modelling and data inputs/outputs GAMS (General Algebraic Modelling System), a high level language, was used [20]. The 
Modified OA/ER algorithm and the two-phased optimization were applied, where GAMS/CONOPT2 (Generalized reduced-gradient method) [21] was used to solve NLP subproblems and GAMS/Cplex 7.0 (Branch and Bound) [22] was used to solve MILP master problems.

The optimization model contained 120 (in)equality constraints, 169 continuous and 122 binary variables. The final optimal solution of 122,60 tons was obtained in the $4^{\text {th }}$ main MINLP iteration.

The optimal result represents the mentioned optimal structure mass of 122,60 tons, the obtained optimal topology of 14 portal frames an 14 purlins, see Fig. 2, and the calculated optimal standard sizes of columns, beams and purlins, see Fig. 3.
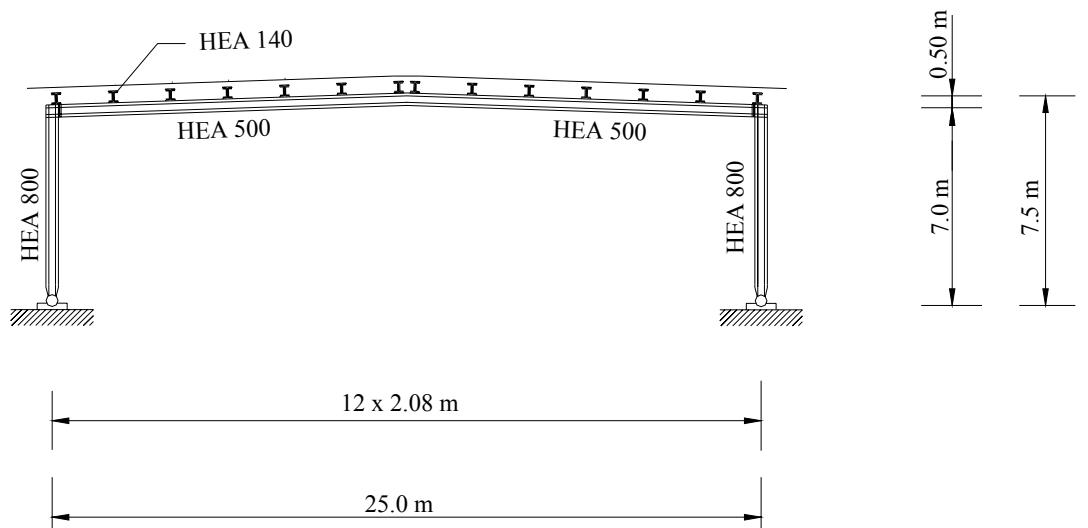

Figure 3: $\quad$ Optimal steel sections.

\section{Conclusion}

The paper presents the Mixed-Integer Non-linear Programming approach (MINLP) to structural optimization. The Modified OA/ER algorithm and the two-phase MINLP optimization strategy were applied. The optimization is performed by a user-friendly version of the MINLP computer package MIPSYN. Beside the optimal structure costs or mass, the optimal topology with the optimal number of structural elements, the optimal discrete/standard cross-sectional sizes are obtained simultaneously. The example, presented at the end of the paper, clearly show the efficiency of the proposed MINLP approach.

\section{References}

[1] E.J. O'Brien, A.S. Dixon, Optimal plastic design of pitched roof frames for multiple loading, Comput. Struct. 64, 737-740, 1997.

[2] G. Gurlement, R. Targowski, W. Gutkowski, J. Zawidzka and J. Zawidzki, Discrete minimum weight design of steel structures using EC3 code, Struct. Multidisc. Optim. 22, 322-327, 2001. 
[3] Eurocode 3, Design of steel structures, European Committee for Standardization, 1992.

[4] M.P. Saka, Optimum design of pitched roof steel frames with haunched rafters by genetic algorithm, Comput. Struct. 81, 1967-1978, 2003.

[5] S. Hernández, A.N. Fontán, J.C. Perezzán, P. Loscos, Design optimization of steel portal frames, Adv. Eng. Software. 36, 626-633, 2005.

[6] Kravanja, Z. and Grossmann, I.E., New Developments and Capabilities in PROSYN - An Automated Topology and Parameter Process Synthesizer, Computers chem. Eng., 18, 1097-1114, 1994.

[7] Kravanja, S., Kravanja, Z. and Bedenik, B.S., The MINLP optimization approach to structural synthesis. Part I: A general view on simultaneous topology and parameter optimization, Int. J. Numer. Methods Eng. 43, 263292, 1998.

[8] Kravanja, S., Kravanja, Z. and Bedenik, B.S., The MINLP optimization approach to structural synthesis. Part II: Simultaneous topology, parameter and standard dimension optimization by the use of the Linked two-phase MINLP strategy, Int. J. Numer. Methods Eng. 43, 293-328, 1998.

[9] Beale, E.M.L., Integer Programming, The State of the Art in Numerical Analysis, ed D. Jacobs, pp. 409-448, Academic Press, London, 1977.

[10] Gupta, O.K. and Ravindran, A., Branch and bound experiments in convex nonlinear integer programming, Management Science, 31(12), 1985, pp.1533-1546.

[11] Olsen, G.R. and Vanderplaats, G.N., Method for Nonlinear Optimization with Discrete Design Variables, AIAA Journal, 27(11), 1989, pp. 15841589.

[12] Bremicker, M., Papalambros, P.Y., and Loh, H.T., Solution of MixedDiscrete Structural Optimization Problems with a New Sequental Linearization Method, Computers \& Structures, 37(4), 1990, pp. 451-461.

[13] Westerlund, T. and Pettersson, F., An extended cutting plane method for solving convex MINLP problems, European Symposium on Computer Aided Process Engineering-5, Supplement to Computers Chem. Engng., Bled, Slovenia, 1995, pp. S131-S136.

[14] Benders, J.F., Partitioning Procedures for Solving Mixed-variables Programming Problems, Numerische Mathematik, 4, 1962, pp. 238-252.

[15] Geoffrion, A.M., Generalized Benders Decomposition, Journal of Optimization Theory and Applications, 10(4), 1972, pp. 237-260.

[16] Kocis, G.R. and Grossmann, I.E., Relaxation Strategy for the Structural Optimization of Process Flowsheets, Ind. Engng Chem. Res., 26, 1987, pp. 1869-1880.

[17] Mawengkang, H. and Murtagh, B.A., Solving Nonlinear Integer Programs with Large-Scale Optimization Software, Annals of Operations Research, 1986, 5, pp. 425-437.

[18] Quesada, I. and Grossmann, I.E., An LP/NLP Based Branch and Bound Algorithm for Convex MINLP Optimization Problems, Computers Chem. Engng., 16, 1992, pp. 937-947. 
448 High Performance Structures and Materials IV

[19] Kravanja, S., Kravanja, Z., Bedenik, B.S. and Faith, S., Simultaneous Topology and Parameter Optimization of Mechanical Structures, Proceedings of the First European Conference on Numerical Methods in Engineering, Brussels, Belgium, ed. C. Hirsch et al., Elsevier, Amsterdam, pp. 487-495, 1992.

[20] Brooke, A., Kendrick, D. and Meeraus, A., GAMS - A User's Guide, Scientific Press, Redwood City, CA, 1988.

[21] Drudd, A.S., CONOPT - A Large-Scale GRG Code, ORSA J. Comput. 6, 207-216, 1994.

[22] CPLEX User Notes, ILOG inc. 